A-26-2020

\title{
OPTIAQUA: HERRAMIENTA PARA GESTIONAR LOS RIEGOS
}

\author{
Huete Cuevas, J. $(P)^{1}$, Tobar Pardo, V. ${ }^{1}$, Omaña Álvarez, J.M. ${ }^{2}$, \\ Quijano García González, A. $^{3}$ \\ 1 Ingeniero Agrónomo, SIAR- Consejería Agricultura - Gobierno de La Rioja, \\ Finca La Grajera. Ctra. LO-20 - salida 13. Autovía del Camino de Santiago, \\ siar.cida@larioja.org \\ 2 Ingeniero Agrónomo, AIMCRA, Ctra. Villabáñez, 201. 47017 Valladolid, \\ j.m.omana@aimcra.es \\ ${ }^{3}$ Ingeniero Agrónomo, Comunidad de Regantes sector $3^{\circ}$ tramo III canal margen \\ izquierda río Najerilla, Apdo. de correos 39, 26200 Haro, \\ secretaria@reganteszarraton.es
}

\section{Resumen}

El método del balance hídrico es una herramienta contrastada para gestionar el riego de las parcelas agrícolas. Sin embargo, su uso no está extendido debido fundamentalmente a la dificultad de acceso en tiempo real a la información necesaria para realizar su cálculo. A través de un Grupo Operativo se ha desarrollado un sistema capaz de calcular los balances hídricos de parcelas de la Comunidad de Regantes de mayor extensión de La Rioja empleando para ello los datos recogidos por los contadores de agua, la información climática de la red de estaciones agroclimáticas de la Rioja y los datos de cultivo recopilados por en distintos trabajos desarrollados por la Consejería de Agricultura de LA Rioja y AIMCRA. EI resultado es una aplicación que permite a los agricultores acceder a través de su teléfono móvil a los resultados del balance hídrico para cada una de sus parcelas y contar así con una referencia adicional a la hora de tomar decisiones de riego.

\section{1- Introducción}

Es la era de la digitalización, se ha puesto de moda la comunicación, la velocidad en las conexiones hace realidad la capacidad para encontrar cualquier tipo de información, en cualquier parte y en unos segundos, escribiendo sobre una pantalla móvil.

La información nos rodea, más bien nos sobrepasa, y aunque casi toda la información actual está digitalizada no toda es accesible vía internet. Mucha de la información que necesitamos está dispersa, por ejemplo necesitamos conocer cuánto ha llovido para programar nuestro sistema de riego, pero también necesitamos conocer cuánto ha consumido nuestro cultivo, comprobar cuál es el estado hídrico del suelo y tener en cuenta la previsión de los próximos días.

Considerando esta abundancia de información, ¿sería posible calcular de una manera rápida y eficaz cuántas horas tengo que regar mis parcelas? Para ello necesitaríamos un método de cálculo contrastado, sencillo y que pudiera ejecutarse de forma automática; tal método existe, se conoce con el nombre de método del balance hídrico y, por lo tanto, la respuesta es sí. Sin embargo, la dificultad se encuentra en la recopilación de la información, 
que debido a su dispersión requiere de una gran cantidad de mano de obra que en la práctica hace imposible el uso de este tipo de herramientas por parte del regante.

\section{2- Descripción y objetivos}

A raíz de este análisis se formó un Grupo Operativo (GO) compuesto por la Comunidad de Regantes sector $3^{\circ}$ tramo III canal margen izquierda río Najerilla, Asociación de Investigación para la Mejora del Cultivo de la Remolacha Azucarera (AIMCRA) y el Servicio de Información Agroclimática de La Rioja que, con financiación de la Unión Europea y del Gobierno de La Rioja, ha trabajado desde 2016 con el objetivo de desarrollar una serie de aplicaciones informáticas que: automaticen la recogida de la información necesaria, ejecuten el cálculo de balance siguiendo las directrices que establece la FAO y muestren al agricultor información veraz y actualizada, de forma sencilla e intuitiva.

OPTIAQUA es la aplicación informática desarrollada en el proyecto de este GO; consta de dos partes, una encargada de recopilar la información de partida y calcular el balance hídrico y otra que, a partir de los datos calculados, ofrece una salida gráfica con el resultado de los balances.

La parte encargada de realizar los cálculos se instala en el servidor de la Comunidad de Regantes y se configura para acceder a los datos climáticos de estaciones meteorológicas públicas cercanas a las zonas de cultivo, así como a los parámetros que definen el suelo de las parcelas (textura, profundidad y porcentaje de elementos gruesos); además, se configura un acceso a la información del riego que puede provenir de la propia comunidad de regantes. Una vez configurada, la aplicación calcula automáticamente los balances para las parcelas que estén activadas en el sistema y ofrece esta información a través de internet.

Por otro lado, la aplicación de visualización de los datos se ejecuta en los teléfonos móviles, tabletas u ordenadores de los usuarios. Mediante un usuario y una contraseña los regantes acceden a la información disponible para sus o unidades de cultivo, llamadas así en lugar de parcelas para distinguirlas de las notación catastral. En la actualidad el sistema OPTIAQUA se ha implantado en la Comunidad de Regantes del sector III, tramo tercero canal margen izquierda del Najerilla y permite a los agricultores adheridos al programa recibir en sus teléfonos móviles la información sobre el estado hídrico de sus unidades de cultivo calculada en tiempo real y, por tanto, tomar decisiones de riego basadas en esta información.

\section{3- Puesta en marcha}

La Comunidad de Regantes cuenta con 4.705 regantes y propietarios declarados, cada año se declaran entre 1400 y 1700 unidades de cultivo, definidas como una entidad homogénea que se puede gestionar integralmente (en lo relativo a riegos y tratamientos) si bien puede contar con una o más posturas de riego. En 2020 se han declarado un total de 788 unidades de cultivo que ocupan una superficie regable de 2.411 hectáreas; la superficie media de cada unidad de cultivo es de 3,06 hectáreas.

El usuario objetivo hacia el que se orienta la herramienta es el agricultor profesional que maneja un gran número de hectáreas con distintos tipos de cultivo; hasta la fecha su sistema de riego es por calendario, con una regulación de las aportaciones de riego basada en la interpretación que los agricultores hacen de la climatología y el estado del cultivo si bien estos cálculos no se realizan siguiendo una metodología estandarizada; estudios previos a este proyecto, realizados entre 2012 y 2015 han demostrado que el empleo del método del balance permite ahorros de hasta un $20 \%$ en las parcelas para las que se realizó el seguimiento.

La configuración de la aplicación que realiza los cálculos comienza con la declaración de cultivos que realizan los agricultores al comienzo de la campaña, en ella se definen las parcelas catastrales que integran cada unidad, el hidrante o hidrante y tomas desde las que se va a obtener el agua de riego y el cultivo que se ocupará cada unidad. A esta información 
que define la superficie de cultivo se le añaden los valores medios de textura del suelo que, junto con el porcentaje de elementos gruesos, permite calcular la capacidad de retención de agua del suelo, para ello se cuenta con los análisis de suelo que realizan los agricultores o bien se puede usar la extensa base de datos de suelo con la que cuenta AIMCRA. Los datos de consumo teórico de los cultivos, que permite asignar una Kc a cada fase de cultivo o, si se dispone de ellas, modelizar el crecimiento del cultivo y obtener el valor de la Kc a partir de los valores de cobertura, están previamente almacenados en distintas tablas de la base de datos, para esta información se ha contado con la información generada por la Consejería de Agricultura de La Rioja que mediante ensayos realizados por la desaparecida sección de Recursos Naturales desde 1980 hasta 2015 caracterizó los cultivos más importantes de la zona.

Por otro lado, para poder realizar los cálculos es necesario acceder, en tiempo real a la información climática y a los volúmenes de riego aplicados. Para lo primero la aplicación accede a la información generada por la red de estaciones del Servicio de Información Agroclimática de La Rioja, este acceso es posible gracias al sistema API con el que cuenta este servicio. En cuanto al riego, se ha generado un sistema de intercambio de información que permite captar los datos recogidos por los contadores de la Comunidad de Regantes.

Los cálculos del balance que permiten obtener los valores concretos de humedad del suelo se realizan siguiendo las directrices especificadas por la monografía 56 de la FAO. La aplicación está desarrollada siguiendo una filosofía de código abierto y está disponible para su estudio o para ser usada como punto de partida en otros proyectos en: https://github.com/OptiAquaProject/OptiAqua

En cuanto al acceso a los datos de balance calculados se ha desarrollado la App OptiAqua. Es una aplicación multiplataforma por lo que se puede acceder a ella tanto desde un dispositivo móvil como desde un ordenador.

Durante el desarrollo de esta aplicación se ha considerado que un regante tiene, por lo general, múltiples unidades de cultivo, cada una con una problemática distinta y en una situación hídrica diferente, por ello tras la pantalla de acceso mostrada a la izquierda de estas líneas donde el regante introduce un usuario y una contraseña se accede a una pantalla inicial donde se muestra el estado hídrico calculado para las unidades de cultivo a día de hoy. Para representar el estado hídrico se utiliza un código de colores que varía entre el verde (estado hídrico OK) al rojo (estado hídrico muy deficitario), ver figura 2. La pantalla inicial permite ordenar las unidades de cultivo en función de distintos criterios: por cultivo, por municipio

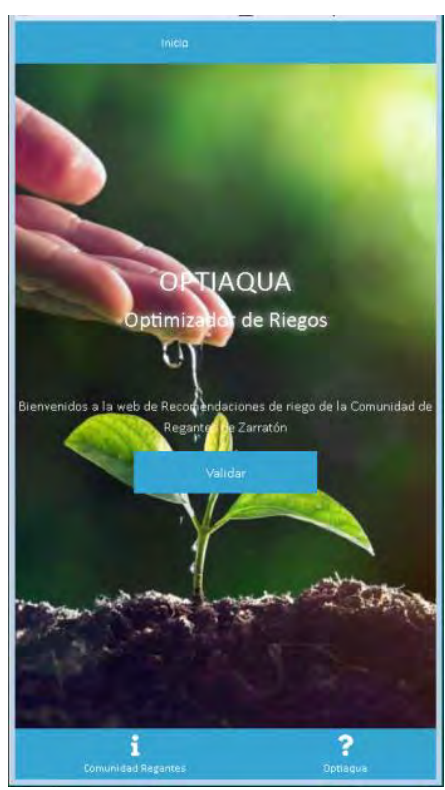
o por el estado hídrico. También se ha pensado en una visión cartográfica de la situación hídrica. 


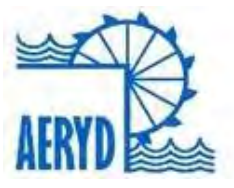

\section{Congreso Nacional de Riegos, Parte I \\ CARTAGENA (Murcia) 2020}

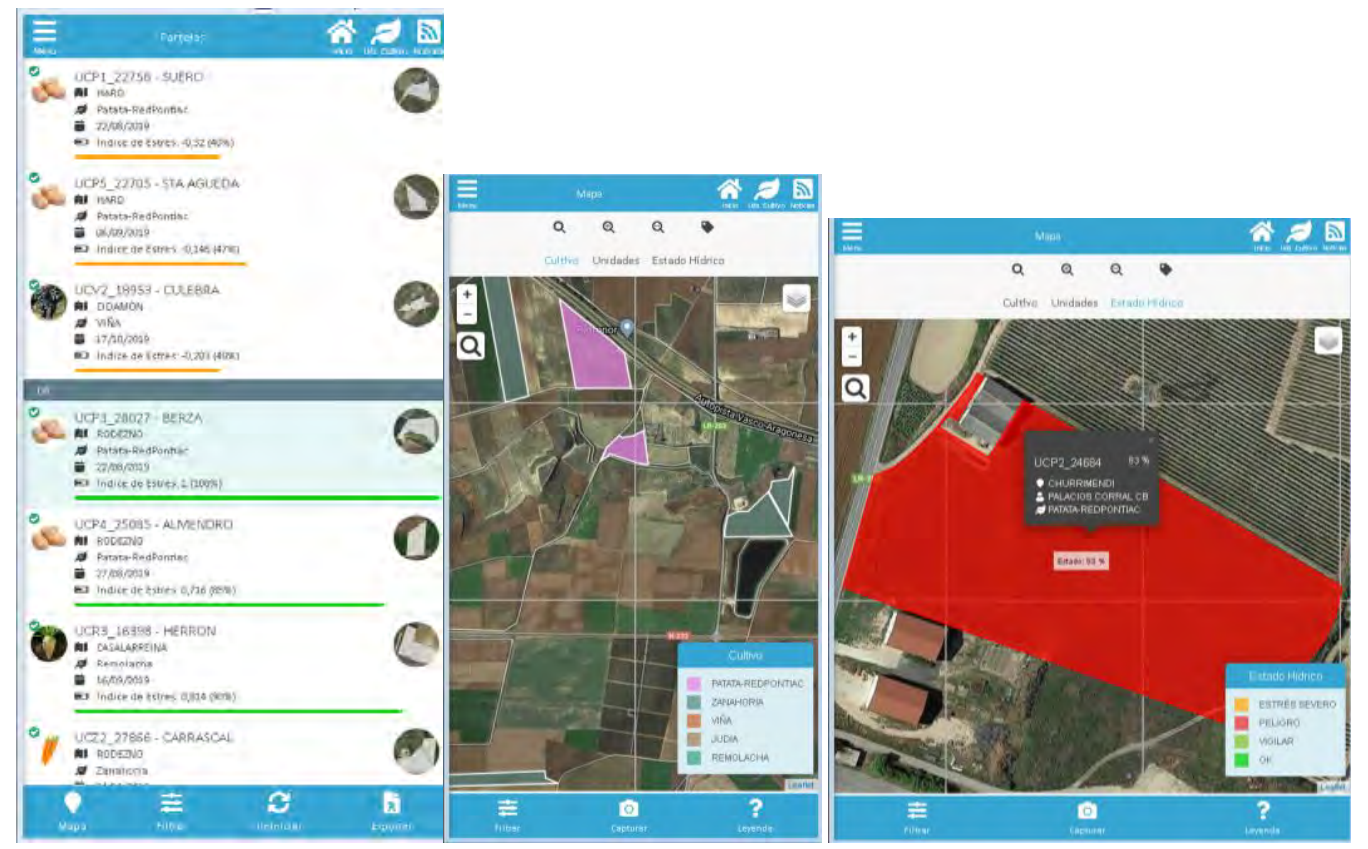

Figura 2. Pantallas iniciales de la aplicación que muestran las parcelas y usnado un código de colores el estado hídrico actual de las mismas.

Tras esta visión general el regante puede acceder a la información pormenorizada para cada una de sus unidades de cultivo, para ello se han diseñado dos pantallas, la más importante es una pantalla donde un cilindro, que representa la capacidad de almacenamiento de agua del suelo, muestra el contenido de agua en el suelo calculado mediante el balance. Esta pantalla se ha definido como una fotografía que refleja el estado actual del cultivo, por ello, además del cilindro, que varía su color en función del estado hídrico de la unidad de cultivo, aparece información adicional relevante para el agricultor como la cantidad de agua total aportada durante la campaña, la cantidad de agua perdida por drenaje y su traducción a euros de pérdidas y las variables de cultivo que se han empleado para realizar los cálculos: fase de cultivo, porcentaje de cobertura, altura del cultivo.

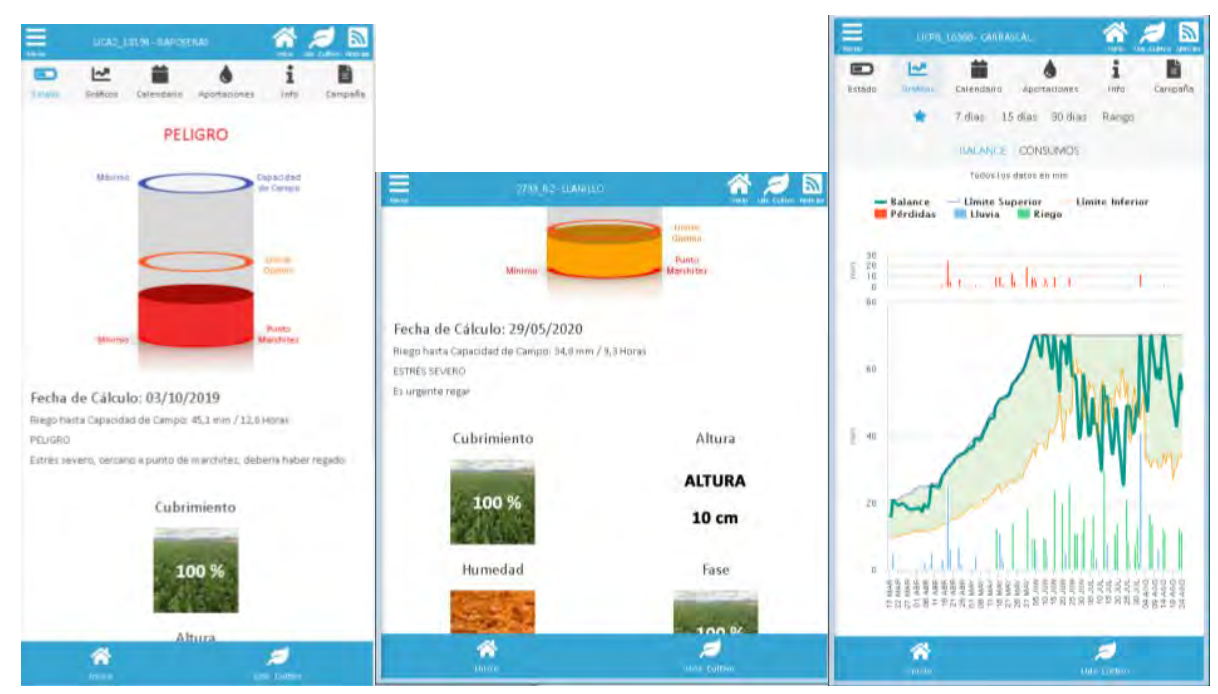

Figura 3: pantallas de detalle (izquierda y centro)donde se muestra tanto el estado hídrico del cultivo como los distintos parámetros empleados en el cálculo y un resumen del agua aportada 
en riego y el agua perdida por drenaje. En la derecha gráfica de evolución del balance en el tiempo que permite un análisis de la estrategia de riego.

Por otro lado se ofrece la clásica salida de balance hídrico (figura 3) representada en una gráfica de evolución que permite analizar para cada día del cultivo el estado hídrico, el consumo diario del cultivo, el riego aportado, las pérdidas por drenaje, etc. Esta pantalla está orientada más a un análisis a posteriori de la situación a realizar por un asesor de riego para evaluar la gestión del riego realizada.

\section{4- Conclusiones}

Mediante este proyecto se ha desarrollado un sistema de aplicaciones informáticas en código abierto que permite el cálculo del balance hídrico y la visualización de los datos por parte del regante o asesor. La arquitectura de ambas aplicaciones y el hecho de estar desarrolladas en código abierto permite adaptarlas a otras comunidades de regantes.

La principal dificultad encontrada ha sido la accesibilidad a la información necesaria para ejecutar los cálculos de balance y ofrecer resultados en tiempo real. Así, si bien esta información está disponible, proviene de distintas fuentes (servicios de información agroclimática, comunidades de regantes, información offline como los análisis de suelo, etc); y se almacena con fines diferentes al de ejecutar un balance hídrico por lo que requiere de un procesamiento previo para ser almacenada en una base de datos unificada. Por ello el principal reto para este tipo de desarrollo ha sido facilitar el entendimiento entre las distintas fuentes de información y su implantación en diferentes organismos o empresas.

Otro problema encontrado y que es específico del territorio en el que nos encontramos, es el reflejo de la realidad territorial en la base de datos. Debido a la singularidad del manejo territorial no es posible automatizar en su totalidad la diversidad de configuraciones de riego y parcelas que existen en la comunidad de regantes.

Por último, a partir del trabajo realizado, se plantean nuevos retos a este GO con la finalidad de mejorar el sistema en la línea de conseguir una mayor precisión y automatización, uno de los más importantes es el empleo de imágenes satélite que nos aporten la información de desarrollo del cultivo, en particular el coeficiente de cultivo (Kc), lo que nos permitiría introducir en el cálculo del balance este valor como una variable medida y no estimada como hasta ahora. 